\title{
Civilisations
}

Revue internationale d'anthropologie et de sciences

humaines

43-2 | 1996

Problèmes africains contemporains

\section{La décennie mondiale du développement culturel (1988-1997)}

\section{René-Pierre Anouma}

\section{OpenEdition \\ Journals}

Édition électronique

URL : http://journals.openedition.org/civilisations/1574

DOI : 10.4000/civilisations. 1574

ISSN : 2032-0442

\section{Éditeur}

Institut de sociologie de l'Université Libre de Bruxelles

\section{Édition imprimée}

Date de publication : 1 avril 1996

Pagination : 161-180

ISBN : 2-87263-160-7

ISSN : 0009-8140

Référence électronique

René-Pierre Anouma, «La décennie mondiale du développement culturel (1988-1997) 》, Civilisations [En ligne], 43-2 | 1996, mis en ligne le 29 juin 2009, consulté le 19 avril 2019. URL : http:// journals.openedition.org/civilisations/1574; DOI : 10.4000/civilisations.1574

Ce document a été généré automatiquement le 19 avril 2019

(c) Tous droits réservés 


\title{
La décennie mondiale du développement culturel (1988-1997)
}

\author{
René-Pierre Anouma
}

\section{Introduction}

1 L'analyse des déséquilibres qui caractérisent la situation mondiale fait apparaître que l'économie ne peut plus constituer l'unique terme de référence en matière de développement. Et si la coopération pour le développement manque d'efficacité, constatent certains experts, cela tient très souvent au fait que les facteurs culturels sont négligés et d'insister, ces mêmes experts, sur la nécessité de tenir compte de l'environnement social et culturel des pays bénéficiaires.

2 De nombreux projets de développement exécutés dans les pays du tiers monde ont, en effet, connu un échec total ou partiel. Le bilan général en ce qui concerne l'Afrique est largement négatif. C'est le constat qu'en ont fait les chefs d'Etat et de gouvernement qui ont affirmé dans le Plan d'action pour le développement économique de l'Afrique que "les effets des promesses non réalisées des stratégies globales de développement ont été plus profondément ressentis en Afrique que dans les autres continents du monde..."1.

3 La prise en compte des facteurs socioculturels aurait pu, pense-t-on aujourd'hui, permettre à défaut d'éviter ces échecs répétés, d'en atténuer au moins les effets pervers. $C^{\prime}$ est dire que le développement possède une dimension culturelle à prendre en compte dans la conception et la mise en oeuvre des projets de développement. L'admettre, c'est reconnaître à la culture une dimension essentielle dans la vie de chaque individu et de chaque communauté et la considérer comme un facteur déterminant de progrès.

4 Réaffirmer la place de la culture dans la société et développer chez les décideurs et les responsables la conscience de la nécessité de la prise en compte de la dimension culturelle dans le développement, notamment dans les domaines qui font l'objet d'une planification d'Etat, ont figuré au nombre des préoccupations majeures qui ont inspiré la décision prise par la communauté internationale d'instituer une Décennie mondiale du développement culturel. 
5 En effet recommandée par la Conférence mondiale sur les politiques culturelles (Résolution 27, Mondialcult, Mexico 1982), la Décennie mondiale du développement culturel a été proposée par la $23^{e}$ session de la Conférence générale de l'UNESCO (Résolution 23 C/11-10 Sofia, octobre-novembre 1985). Elle a, par la suite, été adoptée par la $41^{\mathrm{e}}$ session de l'Assemblée générale des Nations unies le 8 décembre 1986 (Résolution 41/187) qui a proclamé la période 1988-1997, Décennie mondiale du développement culturel.

6 Cette résolution en a défini les objectifs fondamentaux tandis que les modalités de sa mise en oeuvre étaient précisées lors de son lancement le 21 janvier 1988 par le secrétaire général des Nations-unies et le directeur général de l'UNESCO².

7 Les objectifs de la Décennie - prise en considération de la dimension culturelle du développement, affirmation et enrichissement des identités culturelles, élargissement de la participation à la vie culturelle et promotion de la coopération culturelle internationale - supposent la mise en oeuvre d'activités conduites avec le concours des États membres, des organisations intergouvernementales, internationales, et toutes les communautés scientifiques et culturelles aussi bien dans les domaines de la culture que ceux de l'éducation, de la communication, des sciences exactes et naturelles, des sciences sociales et humaines et à travers des projets multisectoriels ou transdisciplinaires.

8 Ainsi de par les compétences que lui confère son Acte Constitutif, l'UNESCO a-t-elle été chargée, après l'avoir initiée, de conduire la Décennie.

Nous nous proposons dans cette réflexion d'en retracer l'évolution et la maturation à travers les différentes conférences intergouvernementales qu'elle a organisées.

De Venise en 1970 à Mexico en 1982, la Décennie apparaît bien comme le résultat d'un long processus de réflexion menée par l'ensemble de la communauté internationale.

11 Ces Conférences régionales, il y en a eu cinq, ont cristallisé la prise de conscience de la place éminente qui revient à la culture dans le monde moderne. Elles devaient, en outre, jouer un très grand rôle dans l'émergence et l'enrichissement d'idées-forces telles que le développement culturel, la dimension culturelle, le développement endogène et de participation de la population à la création de sa culture. Les responsabilités qui incombent aux États à l'égard de la vie culturelle des nations n'en ont été que plus clairement exprimées et mieux définies.

\section{Eurocult ou la Conférence intergouvernementale sur les politiques culturelles en Europe (Helsinki, 19-28 juin 1972)}

12 La Conférence d'Helsinki, la première de la série des réunions régionales organisées conformément aux recommandations de Venise s'est tenue du 19 au 28 juin 1972².

Pourquoi l'Europe d'abord? A ceux qui pourraient s'interroger voire s'en offusquer, René Maheu alors directeur général de l'Unesco demande de ne point "l'interpréter comme la reconnaissance d'une primauté culturelle" qui pourrait d'ailleurs se justifier, selon lui, par "le rayonnement", voire "la dénomination que des facteurs extra-culturels-techniques, militaires, politiques, économiques - ont assuré pendant plusieurs siècles à la civilisation européenne à travers le monde"4. Il explique cette primauté, et nous noterons au passage l'euro-péocentrisme, par le fait que "l'Europe peut légitimement s'enorgueillir d'avoir donné naissance à des cultures à 
vocation universelle dont la fécondité ne cesse, aujourd'hui encore, de se manifester bien au-delà de ses frontières"4.

14 Analysant l'état et les tendances des politiques culturelles en Europe, la Conférence d'Helsinki devait reconnaître "unanimement que le développement culturel fait partie intégrante du développement global et que la politique culturelle représente un facteur essentiel du développement socio-économique de chaque nation"5. Il était, en effet, apparu aux participants que "quelles que soient les structures politiques et socio-économiques des pays concernés, la culture, prise dans son acception la plus large, est devenue de nos jours une composante inséparable de la vie de l'homme et qu'en s'organisant la culture peut accroître son impact sur la vie moderne et sa capacité d'accélérer le progrès général de l'humanité" ${ }^{5}$.

15 Par rapport à Venise qui fut, de l'avis de la Conférence, la formulation "de voeux et d'espérances", mais qui eut le mérite de proclamer que le développement culturel est une dimension essentielle du développement intégral, Helsinki a, en revanche, produit des idées qualifiées par René Maheu comme "autant de clés pour ouvrir les portes de l'avenir"4.

Ces idées reflétées dans les différentes recommandations faites par la conférence concernaient la multiplicité des conceptions nouvelles relatives à l'analyse des problèmes, ceux en particulier du développement et de la société, les nouvelles orientations de la recherche voire certaines esquisses de solutions.

17 La Recommandation $\mathrm{n}^{\circ} 1$, par exemple, reconnait que "la croissance économique règle les efforts des sociétés modernes et constitue pour de nombreux pays une nécessité essentielle". Mais elle fait aussi remarquer que "cette croissance entraîne des déséquilibres qui se manifestent surtout par une inadaptation de plus en plus accusée de l'homme à son milieu de vie, par une progression quantitative proposée comme objectif premier alors que le développement d'une société devrait tendre à l'amélioration qualitative de la vie"6.

18 Face à ce constat, l'élargissement de la notion de culture impose aux gouvernements, audelà de la reconnaissance des droits culturels, la nécessité d'en assurer le plein exercice par l'élaboration et la mise en oeuvre de politiques culturelles dont les finalités vont, elles aussi, au-delà du développement culturel pour créer une culture de développement. Aux termes mêmes de la Recommandation, c'est cette culture de développement qui doit permettre "aux individus de construire l'avenir de leur société et non de le subir; (de) raccorder chaque homme à son milieu de vie; (de) l'intégrer à sa communauté et (de) développer les possibilités pour chaque homme de librement s'exprimer, communiquer, créer"6.

19 Ces conditions devraient permettre, parce que le développement culturel est aussi le "complément et le correctif qualitatif du développement en général", de "restituer à l'homme un milieu naturel et un cadre de vie qui perpétuent la nature et permettent la vie"6. Cette nécessité impose en particulier aux États européens dont le continent fut, de l'aveu de Maheu, " dans le passé le théâtre de guerres dévastatrices" ${ }^{15}$, des responsabilités particulières.

La Conférence devait d'ailleurs leur recommander de promouvoir des actions qui, à travers la variété des démarches et des politiques culturelles, les réunissent non seulement entre eux mais les unissent aux autres pays membres. La Recommandation $\mathrm{n}$ - 21 rappelle à ce propos que "la coopération des États européens doit avoir pour but le renforcement de la peux et la sécurité internationale"7.

21 Ces objectifs répondent aux intérêts de tous les peuples du monde et facilitent plus particulièrement le progrès culturel général des pays en développement. La même Recommandation demande aussi "d'accorder aux pays en voie de développement l'aide nécessaire pour l'exécution des projets pilotes les plus importants dans le domaine de la culture, 
compte tenu des besoins essentiels de ces pays (mais) en respectant toujours le caractère original et unique du patrimoine commun de l'humanité"' .

Helsinki aura été, en définitive, la prise de conscience non seulement de la nature et de l'importance des problèmes culturels dans le monde moderne, mais encore du rôle qui incombe, en la matière, à tous les États membres sans distinction de régime politique. De l'avis de René Maheu, la Conférence d'Helsinki a permis de mettre en lumière la nécessité d'une réflexion fondamentale sur la nature, encore très controversée, de la culture dans la vie moderne.

Yogyakarta offrira l'occasion de poursuivre cette réflexion et de répondre, en ce qui concerne l'Asie, à ces préoccupations.

\section{Asiacult ou la Conférence intergouvernementale sur les politiques culturelles en Asie (Yogyakarta, 10-19 décembre 1973)}

Convoquée par la directeur général de l'Unesco conformément à la résolution 18 C/3.321 adoptée par la dix septième session (1972), cette Conférence s'est réunie à Yogyakarta, en Indonésie, du 10 au 19 décembre $1973^{8}$.

La référence à la culture comme dimension essentielle du développement y a été réaffirmée. En effet, l'examen de l'état et des tendances des politiques culturelles en Asie a fait apparaître, constate encore René Maheu, que " sauf peut-être dans les villes, les notions de droit à la culture et de démocratisation de la culture semblent généralement admises en Asie et l'on constate que le principe du développement culturel comme dimension, voire finalité du développement général, est entré non seulement dans les idées généralement admises, mais même dans certaines constitutions nationales".

Facteur d'identité nationale et instrument de transformation sociale, le développement culturel, affirme la Conférence, doit viser à concilier le développement aux exigences de la technique sans pour autant sacrifier ses valeurs propres et ce qui fait l'originalité de sa culture, parce que seule cette démarche permet d'assurer à la communauté nationale un développement authentique ainsi qu'une indépendance véritable et authentique.

René Maheu remarque ainsi que "cette double révision humaniste de la notion de développement et de l'indépendance politique par l'introduction de la culture marque une évolution décisive de la réflexion sur notre civilisation que nous savons désormais que ni les abstractions de l'économie, ni les idées de la politique ne peuvent à elles seules expliquer, ni guider" . Il importe, donc ajoute-t-il, "de poursuivre résolument cette évolution et d'en tirer les conséquences qui s'imposent"10.

Ainsi au-delà de la "simple fascination du dépaysement", l'attrait qu'exerce l'Asie sur les jeunes des pays industrialisés ne peut s'expliquer, pense René Maheu, que parce que ce continent renferme "dans ses traditions profondes des ressources spirituelles - religieuses, morales ou esthétiques - qui peuvent répondre au besoin de vie intérieure qu'éprouve l'homme de la civilisation industrielle où les mutations rapides constituent autant de facteurs d'aliénation culturelle"10.

Dans un tel contexte, "l'action culturelle et éducative se donnent pour objectif de permettre à chacun d'acquérir conscience critique de sa condition, selon ses motivations, ses aptitudes et ses 
aspirations, et de fournir aux hommes les connaissances et les instruments de développement et d'épanouissement personnels qui facilitent leur participation active à la société" ${ }^{11}$.

C'est la culture, pense en définitive la Conférence, qui seule décide des finalités d'un développement endogène intelligibles à la population, acceptées par elle et, par la suite, susceptibles de bénéficier de sa participation active et réfléchie.

31 Considérant donc que le développement doit viser à enrichir la vie humaine en assurant un harmonieux équilibre entre les valeurs matérielles et spirituelles, sociales et individuelles, la Conférence invite les États membres "à formuler leur objectifs économiques et sociaux dans une perspective culturelle plus large et à réaffirmer les valeurs qui favorisent l'édification d'une société vraiment humaine"12.

Accra allait confirmer, pour l'Afrique, ces objectifs.

\section{Africacult ou la Conférence intergouvernementale sur les politiques culturelles en Afrique (Accra, 27 octobre-6 novembre 1975)}

Cette Conférence a été convoquée par le Directeur général de l'Unesco conformément à la résolution $19 \mathrm{C} / 3.221$ adoptée par la dix-huitième session de la Conférence générale $(1974)^{13}$.

Ici encore la reconnaissance du développement culturel comme dimension essentielle du développement intégral a été largement rappelée par les participants. Pour ceux-ci l'homme étant la véritable finalité du développement, la culture, ont-ils affirmé, "doit retrouver une place centrale dans le processus endogène du développement intégral - faut de quoi ce dernier deviendrait le facteur d'une nouvelle alienation"14. Et c'est pourquoi "l'action économique et l'action culturelle doivent être menées de pair, en tenant compte de la réciprocité de leurs effets, renouant ainsi avec la grande tradition de l'humanisme africain"14. Le développement culturel devient ainsi "non seulement le correctif qualitatif du développement mais la finalité du progrès"14.

L'affirmation de l'identité culturelle et de la sauvegarde de l'authenticité dans la perspective de la reconnaissance culturelle de l'Afrique, objets d'une "remarquable convergence de point de vue", de la part des conférenciers furent, en effet, les principales conclusions qui émergèrent de l'examen de l'état et des tendances des politiques culturelles des États membres d'Afrique.

L'affirmation de l'identité culturelle, plus particulièrement considérée comme "l'accomplissement d'un acte libérateur, une arme de combat au service de l'indépendance effective et l'instrument privilégié de l'épanouissement des individus et de développement harmonieux", apparait ici de plus comme "la condition première de l'avènement d'un nouvel ordre mondial fondé sur le droit imprescriptible des peuples à disposer d'eux-mêmes et la reconnaissance de l'égalité absolue et de la dignité de toutes les cultures"15.

Ainsi l'affirmation de l'identité culturelle procède-t-elle de "l'expression volontaire d'une authenticité qui prend racine dans le génie de chaque peuple et dans les valeurs profondes qui le sous-tendent"15. Mieux, cette recherche de l'authenticité passe par un "ressourcement" qui n'est pas "un simple retour aux sources"mais "intègre les réalités et les impératifs du monde moderne". Elle implique, donc, une prise de conscience lucide qui rend à son tour possibles, l'actualisation et le renouvellement des valeurs. 

19 C/4.131 adoptée par la $19^{\mathrm{e}}$ session de la Conférence générale de l'Unesco, avait pour objet de préciser, en ce qui concerne l'Amérique par la $19^{\mathrm{e}}$ session de la Conférence générale de l'UNESCO, avait pour objet de préciser, en ce qui concerne l'Amérique latine et les Caraïbes, le concept de politique culturelle en fonction des problèmes propres aux États membres de la région ${ }^{18}$. Ceci afin de les aider à définir des stratégies de développement culturel en rapport avec les objectifs qu'ils se proposent en matière de développement général, et de faciliter la coopération culturelle aux échelons régional et international. région a permis de dégager une unanimité en ce qui concerne l'héritage culturel commun et le passé historique analogue des pays d'Amérique latine et des Caraibes. Toutes choses qui n'excluent pas la spécificité et la diversité des cultures propres aux peuples de la région, riche mosaïque culturelle dont la pluralité même représente, selon le constat de la Conférence, "l'élément le plus précieux pour la formulation des solutions de grande portée qu'impose à la région une destinée commune"19. pleine souveraineté de l'indépendance et de la dignité dans le respect mutuel le plus absolu" ${ }^{20}$. 
Quant à l'analyse de la dimension culturelle du développement, elle a conduit la Conférence à examiner la relation entre le développement culturel et le développement technologique. Elle en a conclu "qu'une technologie convenablement aménagée doit jouer un rôle extrêmement important dans le développement socio-culturel de la région" 21 et que "seule une politique culturelle ferme, authentique et appliquée à tous les aspects sans exception de l'effort national peut donner à celui-ci une orientation et un contenu humains et le mettre au service du destin national"21.

C'est en effet par "la participation la plus large, devait constater la Conférence, que peuvent être créées les conditions qui permettent à l'individu ou à la collectivité d'appréhender ses problèmes et $d^{\prime} y$ faire face ${ }^{\text {"21. }}$. De là la conclusion qu'un progrès véritable des hommes et des sociétés ne peut être assuré sans références à la culture. Monsieur M'BOW le confirmera en constatant que: "au cours des dernières années, l'expérience a montré qu'en matière de développement, il ne suffit pas pour réussir de disposer des moyens matériels et techniques". Il importe aussi, fera-t-il encore remarquer, que "les individus et les collectivités qui sont les agents du développement soient eux-mêmes convaincus qu'ils en seront les bénéficiaires"22. C'est à cette condition en particulier que, "la croissance économique, qui est certes la clé de tout développement, cesse d'être en elle-même une finalité" pour devenir "un moyen permettant de satisfaire les besoins de tous, y compris celui d'une possibilité d'épanouissement complet"22. Ainsi perçues, politiques culturelles et politiques de développement se confondent ainsi dans la réciprocité de leurs effets" 22 .

Conference a donc considéré que la culture en tant qu'ensemble de valeurs et de création d'une société et expression de la vie même, est essentielle à celle-ci et n'est pas un simple moyen ou un instrument accessoire de l'activité sociale. Elle en conclut qu'un développement authentique doit procéder des virtualités et de la volonté de chaque peuple et des créateurs qu'il compte. Elle demande ici aussi aux organismes de financement de prendre davantage de mesures concrètes en vue d'introduire une dimension culturelle dans leurs projets intéressant les États de la région.

Les recommandations de Bogota reprenaient, en ce qui concerne le développement économique, celles exprimées à Accra. La Conférence de Bagdad organisée par l'Alesco en novembre 1981 allait dans le même sens.

\section{La troisième Conférence des Ministres responsables des Affaires Culturelles pour les pays arabes (Bagdad, 2-5 novembre 1981)}

48 La Conférence intergouvernementale sur les politiques culturelles dans les États arabes s'étant "heurtée à des obstacles qui ont conduit à la reporter sine die", ce fut l'Organisation arabe pour l'éducation, la culture et la science (Alesco), sur les instructions du Conseil de la Ligue des États arabes, qui a établi des contacts avec l'UNESCO afin d'examiner, dans le cadre de l'accord de coopération conclu entre les deux organisations, la question des politiques culturelles dans le monde arabe.

49 Cette question fut inscrite à l'ordre du jour provisoire de la Conférence des ministres arabes de la culture. Son but était de permettre au groupe arabe d'arrêter une position commune sur les problèmes culturels de l'heure et sur lesquels devaient porter les débats de la Conférence de Mexico prévue pour l'année suivante en juillet 1982. 
50 L'occasion était ainsi donnée à la nation arabe conformément à l'ordre du jour, d'apporter sa contribution au mouvement intellectuel et aux expériences scientifiques (...) intervenus dans le monde, au cours de la dernière décennie (1970-1980), pour approfondir et préciser les concepts relatifs à la culture et à son rôle dans le développement de la société ainsi que pour mettre en oeuvre des expériences pratiques visant à concevoir les méthodes les plus appropriées pour donner à l'action culturelle la place qui lui revient parmi les activités de la société" ${ }^{23}$.

51 De l'analyse de l'état et des tendances des politiques culturelles de leurs pays, les conférenciers conclurent que la culture de la nation arabe apparait comme "le fondement de sa personnalité, l'expression authentique de ses aspirations et de ses espoirs et le véritable pilier de son unité d'ensemble"24. Dès lors "la préservation de son patrimoine, sa transmission de génération en génération et son renouvellement"considérés comme "les garants de la cohésion de la nation et de sa capacité de jouer son rôle créateur dans la civilisation humaine", apparaissent comme autant d'impératifs pour une politique culturelle qui se voudrait une "action en profondeur"25. C'est-à-dire une politique qui se rattacherait solidement aux structures sociales et économiques, aux conceptions, aux façons de penser mentales et aux comportements, ainsi que tous les facteurs qui exercent leur influence sur le milieu socioéconomique.

52 En effet, "face aux défis et aux tentatives de dépossession auxquels est soumise la culture arabe et qui visent à lui imposer des modèles étrangers en matière linguistique, intellectuelle et culturelle, à éliminer les traits distinctifs de la personnalité arabe, à inonder la société arabe de productions culturelles contraires aux valeurs authentiques, à diffuser des formes nuisibles de sous-culture, $\grave{a}$ dénaturer l'histoire arabo-musulmane, à modifier la structure sociale et démographique de la société arabe, à dégrader ses biens culturels et à profaner ses lieux saints" ${ }^{26}$, la Conférence a-telle recommandé "d'éliminer la trace de l'aliénation culturelle et de l'invasion intellectuelle sous toutes leurs formes, apparentes ou cachées", résultats "des invasions coloniales subies dans le passé par la nation arabe, et de s'opposer aux formes nouvelles de colonialisme, notamment à l'entreprise sioniste de colonisation"26.

53 Les conférenciers ont, par ailleurs, constaté, à la lumière de leur expérience et de leur réflexion qu'il n'était pas possible de "fragmenter le développement global en secteurs indépendants"et que le développement culturel, demeurait l'une des dimensions naturelles essentielles du développement global et qu'il était, partant, "illogique de dissocier l'approche économique de l'approche culturelle du développement". Trois conclusions essentielles en ont été tirées, à savoir que l'approche purement économique est aujourd'hui dépassée au profit d'une approche globalisante impliquant une inter-action entre les différentes dimensions - économique, sociale et culturelle d'une part, d'autre part les ressources humaines demeurent l'atout le plus précieux d'une société et enfin l'homme est la finalité de tout développement véritable ${ }^{27}$. En effet les transformations économiques qui n'ont pas été accompagnées d'aucune planification culturelle ont largement contribué, ont encore fait observé les conférenciers, à l'éclosion "de problèmes dont souffrent bien de sociétés, à savoir: déviance, aliénation culturelle, schizophrénies, dégradation des relations humaines et rejet des valeurs spirituelles"27.

54 C'est pour éviter cet état de chose dont elle a beaucoup souffert que la société arabe s'oriente progressivement, depuis peu, vers la planification et la programmation d'un développement socio-économique global et directement applicable.

Reste à approfondir cette approche du développement encore théorique et à la traduire concrètement dans les domaines d'action de développement. C'est donc tout 
naturellement que la Conférence de Bagdad devait recommander, au titre du développement culturel et du développement global de "chercher à intégrer les plans de développement culturel dans la perspective d'un développement global et d'en faire l'un des axes essentiels du développement, de manière à parvenir aux buts recherchés et à réaliser l'adéquation entre la culture et la vie économique" 28 .

\section{Conclusion}

D'une Conférence à l'autre se sont ainsi progressivement précisés certains concepts et principes : le développement culturel comme partie du développement général, l'identité culturelle dont l'affirmation et la préservation deviennent une préoccupation légitime voire majeure, le droit à la culture et corrélativement les devoirs des pouvoirs publics de contribuer à y donner plein effet ; la démocratisation de la culture par l'accès aux moyens et la participation toujours plus large à la vie culturelle.

Appelée à dresser le bilan de l'expérience acquise en matière de politiques et de pratiques dans le domaine de la culture, de susciter une réflexion approfondie sur les problèmes fondamentaux de la culture dans le monde contemporain et de formuler des orientations nouvelles propres à renforcer la dimension culturelle du développement général et à faciliter la coopération culturelle internationale, la Conférence de Mexico - étape importante dans l'approfondissement et l'enrichissement des concepts et des principes dégagés au cours des Conférences régionales - recommande, la proclamation d'une Décennie mondiale du développement culture ${ }^{29}$. Pourquoi ?

Cette décision procède d'un constat et postule une nouvelle orientation.

La Décennie traduit en effet la prise de conscience d'une conception restrictive du développement ne valorisant que les critères économiques et scientifiques sans référence, ou à peine, à l'homme; thèmes majeurs des réflexions des différentes conférences, la dimension et la finalité du développement ont permis de mettre en exergue les rapports dialectiques entre culture et développement et de reconnaître que la notion d'identité culturelle se situe au coeur même de la problématique du développement. Car "longtemps identifié à la simple croissance économique linéaire se mesurant par les indicateurs du PNB et s'inspirant d'un évolutionnisme inconscient ou inavoué, le développement s'est révélé, et c'est une des conclusions de Mexico, être un processus infiniment plus complexe, global et multidimensionnel, efficace seulement s'il repose sur la volonté de chaque société elle-même et s'il exprime vraiment son identité profonde" ${ }^{30}$.

C'est dire qu'un véritable développement ne peut être qu'une entreprise suscitée de l'intérieur, voulue et conduite solidairement par l'ensemble de la communauté.

Puisque la croissance économique ne doit plus être une fin en soi, tout développement doit s'articuler autour de l'homme et de son unicité. C'est dire qu'il ne saurait y avoir de développement sans action volontaire de l'homme ; l'homme finalité du développement n'étant rien d'autre que le produit de sa culture.

2 Enraciner l'économie dans la culture, c'est la nouvelle démarche que postule la Décennie.

Parce que le développement économique qui ignore les réalités socioculturelles induit des effets pervers et suscite de graves problèmes sociaux, la nécessité est donc apparue de concevoir un concept global de développement qui ferait de la société "un ensemble organique dont l'identité culturelle serait la principale force mobilisatrice" ${ }^{13}$. Mais 
comment intégrer la programmation culturelle dans la planification du développement national et comment faire pour que la vie culturelle devienne le point de départ et l'objectif des stratégies de développement, quand on sait, et cela a été reconnu à Mexico, qu'une telle intégration "soulève des problèmes qui n'ont pas toujours reçu de réponse tant pratique que conceptionnelle" ${ }^{132}$ et que "articuler de façon organique les dimensions culturelle et économique d'un développement intégral ne relève pas encore d'une approche théorique rigoureuse " ?32.

L'action de l'UNESCO apparait, dans ces conditions, particulièrement décisive puisqu'il lui revient, et ce fut le voeu exprimé à Mexico, "de faire progresser la recherche tant sur les indicateurs socioculturelles que sur les méthodologies qualitatives d'évaluation applicables aux activités culturelles" ${ }^{132}$;comme il entre dans ses compétences d'entreprendre des recherches interdisciplinaires conduites dans le sens d'une meilleure connaissance des sociétés et des facteurs endogènes de développement. Toutes choses destinées à faire comprendre aux planificateurs et aux décideurs que "la culture constitue une ressource en elle-même qui peut et doit être utilisée dans le processus de développement" d'une part et d'autre part que "la conscience que la société a de sa culture est un facteur essentiel de développement" 32 .

Ainsi en recommandant une Décennie mondiale du développement culturel, l'UNESCO offre-t-elle aux États membres une occasion pour explorer de nouveaux chemins afin de réviser et de renforcer leurs politiques culturelles, et développer toutes les possibilités qu'offrent la science, la technique, les communications et l'éducation modernes. La Décennie devient l'espoir de l'avènement d'un nouvel état d'esprit favorable au dialogue et à l'action concertée aussi bien des individus, des communautés que des nations ; ce qui devrait permettre à la communauté mondiale de relever solidairement les défis de cette fin de siècle.

\section{NOTES}

1. KOSSOU T. BASILE, La dimension culturelle du développement dans le Plan d'action de Lagos pour le développement économique et social de l'Afrique, in Publi. de l'Institut Culturel Africain pp. 313-322.

2. Unesco-Presses, $n^{\circ}$ 4, 22 janvier 1988.

3. Conférence intergouvernementale sur les politiques culturelles en Europe, Rapport final, Helsinki, 19-28 juin 1972, Unesco, 93 p.

4. Helsinki op cit p. 64 , Annexe III.

5. Helsinki op cit p. 7.

6. Helsinki op cit pp. 21 à 23 , Recommandation $n 1$

7. Helsinki, op cit pp. 42 à 44 , Recommandation 21

8. Conférence intergouvernementale sur les politiques culturelles en Asie, Rapport final, Yogyakarta, 10 - 19 décembre 1973, UNESCO, 92 p.

9. Yogyakarta, op cit p. 59, Annexe III.

10. Yogyakarta, op cit p. 60.

11. Yogyakarta, op cit p. 61.

12. Yogyakarta, op cit p. 20. 
13. Conférence intergouvernementale sur les politiques culturelles en Afrique, Rapport final, Accra, 27 octobre-6 novembre 1975, UNESCO, 107 p.

14. Accra, op cit, p. 9

15. Accra, op cit, p. 7

16. Accra, op cit p. 62 .

17. Accra, pp. 43 à 45, Recommandation n 25 .

18. Intergouvermental Conference on Cultural policies in Latin America and the Caribbean, Final report, Bogota, 10-20 jannary 1978, UNESCO p. 114.

19. Bogota, op cit p. 7.

20. Bogota, op cit p. 5 .

21. Bogota, op cit p. 70

22. Bogota, op cit p. 70

23. Troisième Conférence des ministres responsables des affaires culturelles dans les pays arabes, Rapport final, Bagdad, 2-5 novembre 1981, Alesco 69 p. p. 10.

24. Bagdad, op cit p. 15

25. Bagdad, op cit p. 20

26. Bagdad, op cit p. 43

27. Bagdad, op cit p. 19

28. Bagdad, op cit p. 47

29. Conférence mondiale sur les politiques culturelles, Mexico, 26 Juillet 6 août 1982, Rapport final, UNESCO, $211 \mathrm{p}$.

30. Mexico, op cit, p. 6

31. Mexico, op cit p. 22

32. Mexico, op cit p. 23.

\section{RÉSUMÉS}

On the 8th december 1986 the world Decade of cultural development (1988-97) has been adopted by the General Assembly of United Nations which has been proposed by Unesco in 1985.

Due to its constitution Unesco has to initiate and lead the world Decade. Its goals are cultural development, cultural identity, participation to cultural life and international cultural cooperation.

The article emphasizes on the process that led to the Decade, through the five regional conferences (Europe, Asia, Africa, Latine America and West Indies, and Arabia), that appeared as the result of the reflexion of the entire community.

\section{AUTEUR}

\section{RENÉ-PIERRE ANOUMA}

Université d'Abidjan 